\title{
Methicillin Resistant Staphylococcus Aureus (MRSA) Eradication Prior to Cardiac Surgery
}

\author{
Sanjeet Avtaar Singh ${ }^{1}$, Kasra Shaikhrezai ${ }^{1}$, Rajdev Singh Toor ${ }^{2}$, Ahmed Al-Adhami ${ }^{1}$, \\ Sudeep Das De ${ }^{1}$, Renzo Pessotto ${ }^{3}$ \\ ${ }^{1}$ Department of Cardiothoracic Surgery, Golden Jubilee National Hospital, Clydebank, UK \\ ${ }^{2}$ Department of Anesthesiology, Fiona Stanley Hospital, Murdoch, Australia \\ ${ }^{3}$ Department of Cardiothoracic Surgery, Royal Infirmary of Edinburgh, Edinburgh, UK
}

Email address:

Sanjeet_86@hotmail.com (S. A. Singh)

\section{To cite this article:}

Sanjeet Avtaar Singh, Kasra Shaikhrezai, Rajdev Singh Toor, Ahmed Al-Adhami, Sudeep Das De, Renzo Pessotto. Methicillin Resistant Staphylococcus Aureus (MRSA) Eradication Prior to Cardiac Surgery. International Journal of Cardiovascular and Thoracic Surgery. Vol. 3, No. 3, 2017, pp. 18-22. doi: 10.11648/j.ijcts.20170303.12

Received: June 8, 2017; Accepted: July 11, 2017; Published: August 16, 2017

\begin{abstract}
The problem of methicillin-resistant Staphylococcus aureus (MRSA) infections has led to routine surveillance and decolonisation strategies. Cardiac surgery unit admissions receive MRSA cultures from nares, skin, throat and wounds/groin, and if positive are isolated followed by eradication treatment. This strategy was retrospectively reviewed. The study comprises 50 patients: 32 successfully decolonized/eradicated carriers and 18 unsuccessfully eradicated carriers. A comparison of preoperative characteristics showed no statistically significant differences between the 2 groups with the exception for asthmatic patients, where there was only $3.1 \%$ of MRSA eradicated patients vs $22.2 \%$ in the unsuccessful eradication group ( $\mathrm{p}=0.031$ ). There was no difference between the operative patient data of both groups in hospital mortality, post-operative lengths of stay, ventilation time, post op IABP (intra-aortic balloon pump), post-operative complications, Cerebrovascular accidents and Transient Ischaemic attacks as well as long term complications were not statistically significant. However, there is a statistically significant difference between the use of post-operative antibiotics, with $72.2 \%$ of unsuccessfully MRSA eradicated patients requiring antibiotics postoperatively, compared to $40.6 \%,(\mathrm{p}=0.032)$. Preoperative asthmatics were more likely to fail MRSA eradication/decolonisation. Post operatively MRSA eradication results in the reduction in postoperative antibiotic use.
\end{abstract}

Keywords: MRSA Eradication, Cardiothoracic Surgery, Surgical Site Infection

\section{Introduction}

Colonisation is a pivotal phase in pathogenesis of Methicillin Resistant Staphylococcus aureus (MRSA) infection. Patients undergoing cardiac surgery are particularly susceptible to MRSA infection due to the nature of invasive interventions comprising valve implantation, extracorporeal circulation, insertion of mechanical support devices and prolonged indwelling intravenous lines [1]. Barber et al reported the first instance of MRSA in 1961 [2].

One in five in the general public is persistently colonized with Staphylococcus aureus, commonly in the anterior nares, followed by the throat, perineum and groin [3]. Also $30 \%$ of the general population develops intermittent colonization, with the remainder not susceptible to colonization for reasons unknown. Almost $70 \%$ of invasive $S$. aureus demonstrate resistance, most of them previously colonized with MRSA prior to infection. [4-6]. Evolution of MRSA is known to have occurred by the acquisition of a mobile genetic element $s$ called staphylococcal cassette chromosome mec (SCCmec) which carries the mecA gene that encodes $\mathrm{PBP} 2 \mathrm{a}$ cell wall protein offering resistance to Methicillin as well as ß-lactams [7].

The ultimate aim of MRSA eradication or decolonization is prevention of both transmission and infection. Several guidelines have been set out to attempt eradication of MRSA whilst decolonization of MRSA may reduce 
organism load, which in turn can reduce the risk of transmission and infection in the patient $[8,9]$.

Patients with active infections, chronic skin lesions, urinary catheters, tracheostomy long lines or other medical devices are unlikely to have their MRSA eradicated after topical decolonisation therapy. The objective of this study is to examine if eradication of MRSA is necessary or MRSA suppression/failed eradication is sufficient in prevention of potential complications and infections in MRSA colonized individuals.

$\mathrm{H}_{0}$ There is no significant difference between postoperative outcomes of patients who have undergone successful MRSA eradication and patients with unsuccessful MRSA eradication.

$\mathrm{H}_{1}$ There is a significant difference in post-operative outcome between patients who have been successfully MRSA eradicated and patients who have not been successfully MRSA eradicated.

\subsection{Materials and Methods}

A prospective case series review was undertaken on all patients undergoing cardiac surgery between $1^{\text {st }}$ January 2005 and $31^{\text {st }}$ December 2010 at the Royal Infirmary of Edinburgh. Pre-, peri- and post-operative data were collected during admission as part of a routine clinical practice.

\subsection{Eradication Protocol}

Screening should consist of 3 swabs, one each from the nose, throat and wound (largest wound if there is more than one). Where there is no wound a groin swab should be was taken. Swabs in bacterial transport medium (e.g. Amies) should be used for MRSA screening.

Swab(s) are used directly for moist areas and moistened with sterile saline for dry areas. MRSA decolonization/suppression should be considered for every patient who is found to be MRSA positive- A risk benefit analysis should be done on a case by case basis and decolonization should always be undertaken with patient and clinician agreement. The decision to attempt MRSA decolonization/ suppression ultimately lies with the medical team responsible for the patient, but it is assumed that most MRSA colonized patients will undergo decolonization/suppression unless this is contraindicated or inappropriate (e.g. terminal care).

Systemic antibiotic therapy was defined as treating infection caused by MRSA, where the treatment strategy for decolonization should be advised by a microbiologist.

Decolonization/suppression regimen was commenced for MRSA positive 5 days prior to operation without delaying surgery.

For high risk operations and when surgery was not urgent, decolonization with follow up re-screening was considered.

Patients undergoing surgery who had their MRSA successfully eradicated still received antibiotic prophylaxis.

\subsection{MRSA Decolonization / Suppression Regimen}

a Nose: Mupirocin 2\% nasal ointment applied to the inner surface of each nostril (anterior nares) three times daily for 5 days.

b Throat: Gargle, mouthwash and oral hygiene with chlorhexidine gluconate $0.2 \%$ mouthwash solution twice daily for 5 days.

c Body: Chlorhexidine gluconate 4\% (Hibiscrub) solution as soap/shampoo substitute once daily for 5 days.

\subsection{Re-screening Following MRSA Decolonization}

At least 48 hours after treatment Swabs were collected from nose, throat and largest wound or groin if there is no wound.

Second screening was undertaken on receipt of the first screening negative results and the third screening commenced on receipt of the second screening negative results.

Third and subsequent courses of decolonization are not recommended because of low rate of success, and increased risk of developing resistance.

Recognized complications of MRSA infections were based on Shekar L. C. Reddy et al [10].

Continuous variables are shown as median with 25th and 75th percentiles due to non-normality of data. Categorical data are shown as percentages. Univariate comparisons were made with Chi-square tests or Fisher's exact test.

All statistical analysis was performed using SPSS 19 for Mac, SPSS Inc., an IBM Company.

\section{Results}

There were $50(1.13 \%)$ patients with positive MRSA colonization preoperatively during the study period (of whom) 32 were successfully eradicated with 3 consecutive positive swabs vs. 18 patients who either did not undergo eradication or had unsuccessful eradication.

These patients with associated mortality are detailed in Table 1. Annual carrier status and infection rates are depicted in Figure. 1.

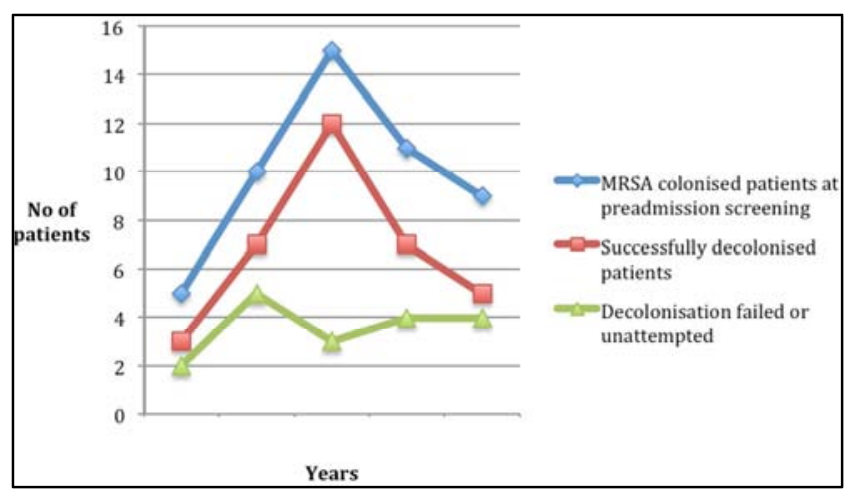

Figure 1. Annual carrier and decolonization status.

Rate of infection in two groups is demonstrated in Figure 2. 


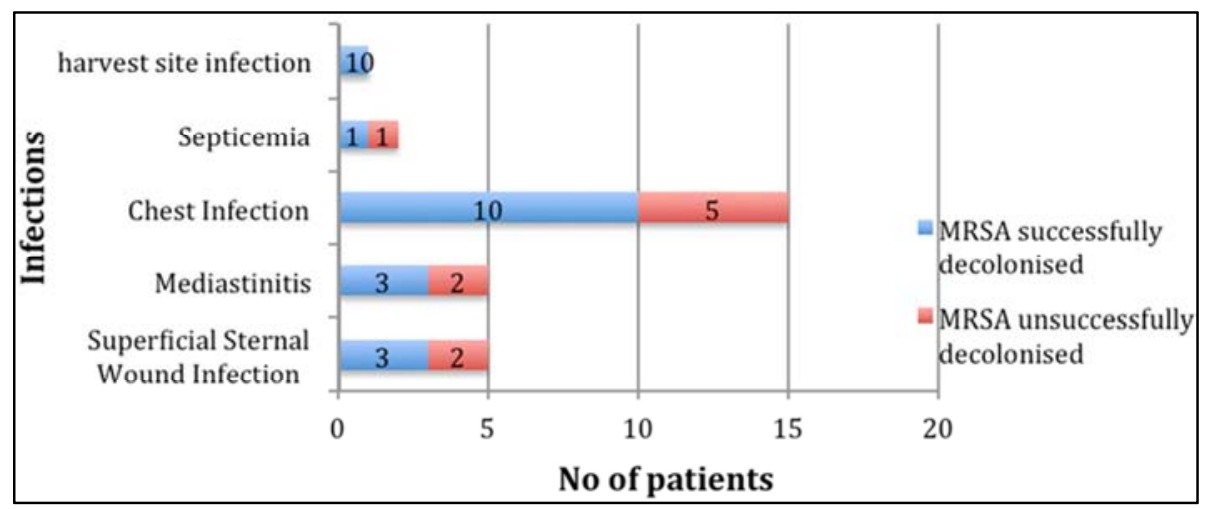

Figure 2. Distribution of Clinical MRSA infections according to preoperative MRSA status.

Table 1. Patients with associated mortality.

\begin{tabular}{lll}
\hline MRSA Status & Number of patients & Percentage In -hospital Mortality \\
\hline All Preoperative Carriers & 50 & 4.0 \\
Successfully eradicated Preoperative carriers & 32 & 3.1 \\
Unsuccessfully eradicated Preoperative carriers & 18 & 5.6 \\
Preoperative carriers with post op surgical infection & 26 & 3.8 \\
Preoperative carriers without post op surgical infection & 24 & 4.0 \\
\hline
\end{tabular}

Table 2. Pre-, peri- and post-operative characteristics.

\begin{tabular}{|c|c|c|c|}
\hline Preoperative variable(s) & Successful Decolonization $(n=32)$ & Unsuccessful Decolonization $(n=18)$ & p-value \\
\hline Age (years) & $71.0(62.3,76.3)$ & $73.0(68.8,73.5)$ & 0.169 \\
\hline $\operatorname{BMI}\left(\mathrm{kg} / \mathrm{m}^{2}\right)$ & $28.2(26.0,30.6)$ & $26.3(23.4,30.3)$ & 0.101 \\
\hline Female $(\%)$ & 37.5 & 27.8 & 0.488 \\
\hline $\mathrm{EF}<30 \%(\%)$ & 12.5 & 0 & 0.118 \\
\hline Diabetes (\%) & 28.1 & 16.7 & 0.361 \\
\hline COPD $(\%)$ & 15.6 & 16.7 & 0.118 \\
\hline Asthma $(\%)$ & 3.1 & 22.2 & 0.031 \\
\hline Current Smoker (\%) & 15.6 & 5.6 & 0.293 \\
\hline Hypertension $(\%)$ & 21.9 & 33.3 & 0.375 \\
\hline PVD $(\%)$ & 18.8 & 33.3 & 0.246 \\
\hline Emergency operation (\%) & 21.9 & 22.2 & 0.977 \\
\hline EuroSCORE & $4.29(2.85,8.23)$ & $4.01(2.81,7.14)$ & 0.742 \\
\hline Peri-operative Variable & MRSA(N=50) Successful Decolonisation & Unsuccessful Decolonisation & p-value \\
\hline Total Bleeding $(\mathrm{ml})$ & $770.0(480,0,1140.0)$ & $790.0(462.5,1160.0)$ & 0.808 \\
\hline $\mathrm{X}$ clamp time (min) & $37.0(0.0,71.3)$ & $33.5(0.0,75.3)$ & 0.324 \\
\hline Bypass time (min) & $88.0(0.0,123.5)$ & $75.5(0.0,120.0)$ & 0.425 \\
\hline Op time (min) & $180(170,241.3)$ & $192.5(140,242.8)$ & 0.385 \\
\hline In hospital mortality (\%) & 3.1 & 5.6 & 0.674 \\
\hline Post Op antibiotic (\%) & 40.6 & 72.2 & 0.032 \\
\hline Renal Failure (\%) & 9.4 & 22.2 & 0.209 \\
\hline CVA/TIA (\%) & 12.5 & 5.6 & 0.432 \\
\hline Ventilation time (mins) & $847.5(521.3,1061.3)$ & $900.0(540.0,1218.8)$ & 0.853 \\
\hline Post Op IABP (\%) & 9.4 & 0.0 & 0.180 \\
\hline Post Op Complications (\%) & 46.9 & 50.0 & 0.832 \\
\hline In hospital stay (hrs) & $142.3(118.8,176.4)$ & $157,8(126.9,239.8)$ & 0.703 \\
\hline Long term complications $(\%)$ & 9.4 & 27.8 & 0.088 \\
\hline
\end{tabular}

Continuous data shown with median values and $25^{\text {th }}$ and $75^{\text {th }}$ percentiles

Categorical data shown as percentages

A comparison of in hospital mortality between the 2 groups revealed no significant difference, $3.1 \%$ vs. $5.6 \%$ $(p=0.674)$. Post operative characteristics are presented on table 1 which did not differ significantly between the two groups. There was a significant difference between the two groups in the usage of antibiotics post-operatively, $72.2 \%$ of unsuccessfully MRSA eradicated patients vs. 40.6\%, $(\mathrm{p}=0.032)$.

\section{Discussion}

According to the findings, there was only a significant difference in postoperative antibiotic usage, which was considerably more in the unsuccessful eradication group. 
Combes et al, reported no difference in outcome between MRSA and MSSA mediastinitis sampling 371 patients with mediastinitis [11]. However Zangrilo et al [12] concluded that there is a strong association between the development of MRSA and mortality following cardiac surgery, with unproven causes and effects. These results highlight the need to minimize the risk of MRSA infections within the cardiac setting as although it carries a small incidence; there is a higher associated mortality rate. It must also be stated however that most studies have shown that non-survivors with MRSA also had more comorbidities and therefore it is arguable whether or not MRSA infections played a part for mortality [10].

Kalra et al and Cho et al both drew similar conclusions that there was an increase in MRSA colonisation among patients in the intensive care setting. The susceptibility to colonisation therefore may also be associated with comorbidities although this remains unproven. [13, 14]

Large scaled double blind randomized controlled studies for MRSA decolonisation have largely been inconclusive with regards to cardiac surgery. Perl et al concluded that there was no significant reduction in rates of all nosocomial infections or those caused by S. aureus. [15] But there was a significant reduction in all nosocomial infections in patients who were nasal carriers. One particular cohort study with conclusive results was by Cimochowski et al [7], showing a significant reduction in overall Surgical Site Infection (SSI) rate in active group, but noted an increment in proportion of wound infections caused by MRSA intranasal Mupirocin treated group compared to the no intervention group.

In Orthopaedic surgery, Kalmeijer et al demonstrated no difference in the rate of superficial surgical site infection however deep surgical site were almost eliminated-by just eradicating MRSA in the anterior nares. Intervention did not result in statistically significant overall reduction in MRSA complications. It is concluded that MRSA eradication is a good preventative method, but should be utilised alongside regular surveillance to ensure MRSA infections are controlled [16].

Suzuki et al, described a significant reduction in chest infections without significant reduction in SSI with no reduction in rates of nosocomial MRSA SSI infections in abdominal digestive surgery [17].

MRSA eradication proved to be of great benefit to patients requiring renal replacement therapy that showed a significant decrease in $S$. aureus infections [18]. Two randomised placebo controlled double blinded trials were conclusive with similar results in these populations. A multicentre randomised control trial may answer some of the inconclusive outcomes with larger samples and a more stringent framework for eradication. Literature has been similar in their reports that Mupirocin appears to be the most commonly used agent for eradication of nasal carriage, with less studies done on the other swab sites [19].

This study demonstrates that asthmatic patients are susceptible of developing resistance to eradication protocol. This can be due to previous frequent usage of antibiotic by asthmatic patients. It also showed no significant difference in the occurrence of the post-operative mortality as well as complications in MRSA eradicated patients vs. MRSA patients.

The major limitation in this study was the small sample size of patients. Ideally 3 separate cohorts evaluating the postoperative outcomes of should have been considered, successful eradication, MRSA suppression and MRSA decolonization not attempted. Other limitations include the assumption that patients had to have had complications that were severe enough to warrant in hospital treatment, and those that were managed in the primary care setting were not included.

\section{References}

[1] Bode LG, Kluytmans JA, Wertheim HF, Bogaers D, Vandenbroucke-Grauls CM, Roosendaal R, et al. Preventing surgical-site infections in nasal carriers of Staphylococcus aureus. N Engl J Med. 2010; 362(1): 9-17.

[2] Barber M. Methicillin-Resistant Staphylococci and Hospital Infection. Postgrad Med J. 1964; 40: SUPPL: 178-81.

[3] Wertheim HF, Melles DC, Vos MC, van Leeuwen W, van Belkum A, Verbrugh HA, et al. The role of nasal carriage in Staphylococcus aureus infections. Lancet Infect Dis. 2005; 5(12): 751-62.

[4] Eurosurveillance editorial t. ECDC publishes 2014 surveillance data on antimicrobial resistance and antimicrobial consumption in Europe. Euro Surveill. 2015; 20(46).

[5] Struelens MJ, Monnet D. Prevention of methicillin-resistant Staphylococcus aureus infection: is Europe winning the fight? Infect Control Hosp Epidemiol. 2010; 31 Suppl 1:S42-4.

[6] Dumitrescu O, Lina G. What is the place of linezolid in the treatment of methicillin-resistant Staphylococcus aureus nosocomial pneumonia and complicated skin and soft tissue infections in Europe? Clin Microbiol Infect. 2014; 20 Suppl 4: $1-2$.

[7] Cimochowski GE, Harostock MD, Brown R, Bernardi M, Alonzo N, Coyle K. Intranasal mupirocin reduces sternal wound infection after open heart surgery in diabetics and nondiabetics. Ann Thorac Surg. 2001; 71(5): 1572-8; discussion 8-9.

[8] Coia JE, Duckworth GJ, Edwards DI, Farrington M, Fry C, Humphreys $\mathrm{H}$, et al. Guidelines for the control and prevention of meticillin-resistant Staphylococcus aureus (MRSA) in healthcare facilities. J Hosp Infect. 2006; 63 Suppl 1:S1-44.

[9] Gemmell CG, Edwards DI, Fraise AP, Gould FK, Ridgway GL, Warren RE, et al. Guidelines for the prophylaxis and treatment of methicillin-resistant Staphylococcus aureus (MRSA) infections in the UK. J Antimicrob Chemother. 2006; 57(4): 589-608.

[10] Reddy SL, Grayson AD, Smith G, Warwick R, Chalmers JA. Methicillin resistant Staphylococcus aureus infections following cardiac surgery: incidence, impact and identifying adverse outcome traits. Eur J Cardiothorac Surg. 2007; 32(1): 113-7. 
[11] Combes A, Trouillet JL, Baudot J, Mokhtari M, Chastre J, Gibert C. Is it possible to cure mediastinitis in patients with major postcardiac surgery complications? Ann Thorac Surg. 2001; 72(5): 1592-7.

[12] Zangrillo A, Landoni G, Fumagalli L, Bove T, Bellotti F, Sottocorna $\mathrm{O}$, et al. Methicillin-resistant Staphylococcus species in a cardiac surgical intensive care unit: a 5-year experience. J Cardiothorac Vasc Anesth. 2006; 20(1): 31-7.

[13] Kalra L, Camacho F, Whitener CJ, Du P, Miller M, Zalonis C, et al. Risk of methicillin-resistant Staphylococcus aureus surgical site infection in patients with nasal MRSA colonization. Am J Infect Control. 2013; 41(12): 1253-7.

[14] Cho OH, Baek EH, Bak MH, Suh YS, Park KH, Kim S, et al. The effect of targeted decolonization on methicillin-resistant Staphylococcus aureus colonization or infection in a surgical intensive care unit. Am J Infect Control. 2016; 44(5): 533-8.

[15] Perl TM, Cullen JJ, Wenzel RP, Zimmerman MB, Pfaller MA, Sheppard D, et al. Intranasal mupirocin to prevent postoperative Staphylococcus aureus infections. N Engl J Med. 2002; 346(24): 1871-7.
[16] Kalmeijer MD, Coertjens H, van Nieuwland-Bollen PM, Bogaers-Hofman D, de Baere GA, Stuurman A, et al. Surgical site infections in orthopedic surgery: the effect of mupirocin nasal ointment in a double-blind, randomized, placebocontrolled study. Clin Infect Dis. 2002; 35(4): 353-8.

[17] Suzuki Y, Kamigaki T, Fujino Y, Tominaga M, Ku Y, Kuroda Y. Randomized clinical trial of preoperative intranasal mupirocin to reduce surgical-site infection after digestive surgery. Br J Surg. 2003; 90(9): 1072-5.

[18] Harbarth S, Dharan S, Liassine N, Herrault P, Auckenthaler R, Pittet D. Randomized, placebo-controlled, double-blind trial to evaluate the efficacy of mupirocin for eradicating carriage of methicillin-resistant Staphylococcus aureus. Antimicrob Agents Chemother. 1999; 43(6): 1412-6.

[19] Mody L, Kauffman CA, McNeil SA, Galecki AT, Bradley SF. Mupirocin-based decolonization of Staphylococcus aureus carriers in residents of 2 long-term care facilities: a randomized, double-blind, placebo-controlled trial. Clin Infect Dis. 2003 ; 37(11): 1467-74. 